\title{
十字架型间二咪唑(鎓)苯的合成及对有机酸的识别
}

\author{
王志周红军胡俊毅游劲松* 高戈*
}

(四川大学化学学院 成都 610065)

\begin{abstract}
摘要 通过 Sonogashira 偶联反应、 $N$-芳基化反应和季铵化反应，我们构筑了一类基于咪唑(鎓)的十字架型荧光分子 $\mathbf{4}$ 和 $\mathbf{5}$, 研究了烷基链以及咪唑和咪唑鎓分别作为电子受体对十字架型分子荧光性质的影响. 其中, 分子 $\mathbf{4 a}$ 具有较高的 苂光量子产率和大的斯托克斯位移; 在不同极性的溶剂中表现出明显的溶致变色性质, 苂光颜色覆盖由深蓝色到黄色 区域; 同时，该分子还具有明显的聚集诱导发光特性. 我们采用数码照相的方法代替繁琐的荧光光谱仪，仅使用单一 该荧光分子在两种有机溶剂中即可通过荧光颜色有效区分 15 种结构类似或 $\mathrm{p} K_{\mathrm{a}}$ 相近的有机酸. 最后, 分子 $4 \mathbf{a}$ 和 $\mathbf{5 a}$ 固 载于硅胶板上通过可逆的荧光颜色变化实现了对 $\mathrm{HCl} / \mathrm{Et}_{3} \mathrm{~N}$ 气体的识别.
\end{abstract}

关键词＼cjkstart咪唑; 咪唑鎓; 十字架型荧光分子; 溶致变色; 聚集诱导发光; 识别

\section{Bisimidazole and Bisimidazolium Cruciforms: Synthesis and Discrimination of Organic Acids}

\author{
Wang, Zhi Zhou, Hongjun $\quad$ Hu, Junyi $\quad$ You, Jingsong* Gao, Ge* \\ (College of Chemistry, Sichuan University, Chengdu 610065)
}

\begin{abstract}
Bisimidazole cruciforms $4 \mathbf{a} \& \mathbf{b}$ based on 1,3-di(imidazol-1-yl)-benzene were synthesized in good yields via the Sonogashira coupling of 1,5-dibromo-2,4-diiodobenzene with 4-ethynyl- $N, N$-dimethylaniline and 4-ethynyl- $N, N$-dibutylaniline followed by the $N$-arylation with imidazole. Further quaternization of $\mathbf{4}$ with iodomethane afforded bisimidazolium cruciforms $5 \mathbf{a} \& \mathbf{b}$. As an example, the didonor- $\pi$-diacceptor cruciform $\mathbf{4 a}$ showed strong solvatochromism that its emission colors varied from deep blue (toluene: $422 \mathrm{~nm}$ ) to yellow (DMSO: $516 \mathrm{~nm}$ ) in different organic solvents. The Stokes shift of 4a in acetonitrile was as large as $148 \mathrm{~nm}$, which could suppress the fluorescence quench caused by reabsorption. The single bond connected multi-aromatic system made cruciform 4a possess aggregation-induced emission (AIE) property. A 12-fold enhancement of the fluorescence was observed for the solution of $\mathbf{4 a}$ in methanol/water $(70: 30, V / V)$ compared with in methanol/water $(50: 50, V / V)$. Taking advantage of the digital photography technology, fifteen carboxylic acids having either similar $\mathrm{p} K_{\mathrm{a}}$ values or similar structures were distinguished well by using bisimidazole cruciform $\mathbf{4 a}$ as the only indicator in two solvents. However, bisimidazolium cruciforms 5 could not discriminate them. All analytes were dissolved in dichloromethane and acetonitrile. A $20 \mu \mathrm{L}$ of dilute stock solution of $4 \mathbf{a}$ was added into $2.0 \mathrm{~mL}$ of each such solution. The solutions were well-mixed and subjected to the UV illumination $(365 \mathrm{~nm})$. Photos were then taken using a digital camera one by one. The square segments representative of the emission colors were cut out and arranged into arrays for naked-eye discrimination. These emission colors were later converted into numerical R/G/B values by using Adobe PhotoShop. The standard deviations $\sigma$ s were calculated for the R/G/B value of the solution of 4 a with each analyte towards those with the other analytes, which represented the degrees of discrimination between two analytes. The standard deviations $\sigma$ 's were calculated for the $\mathrm{R} / \mathrm{G} / \mathrm{B}$ values of the solutions of $\mathbf{4 a}$ with different analytes towards that of the blank solution of $\mathbf{4 a}$, which represented the degrees of the color variation caused by the recognition. Comparing with traditional fluorescence sensing method, the present method is simple, efficient and time-saving. Bisimidazole cruciform 4a emitted solid-state florescence of green-yellow color with a quantum yield of 0.33 while bisimidazolium cruciform $5 \mathbf{a}$ emitted pure red color with a quantum yield of 0.14. They exhibited reversible color change under the UV irradiation when contacted with $\mathrm{HCl}$ and $\mathrm{Et}_{3} \mathrm{~N}$ vapors, indicating they could serve as solid sensors for acid vapors. The fluorescence study also showed that the alkyl chains significantly affect the solid-state fluorescence of bisimidazolium cruciform $\mathbf{5}$.

Keywords imidazole; imidazolium; cruciform fluorophore; solvatochromism; aggregation-induced emission; sensing
\end{abstract}

\section{1 引言}

\section{间二咪唑苯是一个具有 $\Lambda$ 型结构的三芳基共轭骨}

架分子。咪唑季铵化后即得到具有广泛用途的鳌型 (Pincer) 氮杂环卡宾 $(N$-heterocyclic Carbene, NHC) 前

\footnotetext{
*E-mail: gg2b@scu.edu.cn; jsyou@scu.edu.cn
}

Received April 1, 2013; published June 27, 2013.

Supporting information for this article is available free of charge via the Internet at http://sioc-journal.cn.

Project supported by the National Natural Science Foundation of China (Nos. 20902063, 21172159) and the Scientific Research Foundation for the Returned Overseas Chinese Scholars, State Education Ministry (No. 20111568-8-2).

项目受国家自然科学基金(Nos. 20902063, 21172159)和国家教育部留学回国人员科研启动基金(No. 20111568-8-2)资助. 
体 $^{[1,2]}$ ，与过渡金属配位形成的配合物在过渡金属催化 反应中表现出优秀的催化性能. 例如: 与金属铑、铱、 锆形成的配合物可以有效地催化氢胺化反应 ${ }^{[3,4]}$; 与铑 配位后可以催化炔烃的氢硅烷化反应 ${ }^{[5]}$ 等. 咪唑鎓不但 自身能与稀土金属离子配位形成苂光离子液 ${ }^{[6]}$, 其形成 NHC 后再与金属配位也能得到新型发光材料, 如与金 属铂配位后发射强的蓝色固体荧光 ${ }^{[7]}$; 与金属锇形成的 一系列配合物的苂光波长在红色 近红外区域 ${ }^{[8]}$. 另外, 间二咪唑苯可作为构筑大环的骨架单元, 最近我们通过 直接的 $[2+2]$ 环化反应, 获得了一种新型的全共轭四咪 唑鎓刚性大环 ${ }^{[9]}$. 此发射强的蓝色苂光的大环分子带有 四个氢键供体, 有望在阴离子识别领域有优异的表现. 间二咪唑苯本身也可以直接用于构筑新颖的功能性材 料. 例如: 其与金属钴或锌配位可以形成三维的金属有 机框架材料 $(\mathrm{MOFs})^{[10,11]}$; 与锰和铜配位可以构建有机无机杂化配位复合物 ${ }^{[12]}$; 与银配位可以获得一种非消 旋的管状配位聚合物凝胶 ${ }^{[13]}$.

咪唑是一种稳定的五元缺电杂芳环, 具有吸电子效 应. 当两个咪唑基团同时连接在中心苯环上的 1 位和 3 位形成 $\Lambda$ 型分子结构, 不仅拓展了分子的 $\pi$ 共轭体系, 而且这种特殊的构型使得间二咪唑苯可以成为构筑二 维的十字架型交叉共轭分子的骨架 ${ }^{[14]}$. 十字架型苂光 分子具有两个正交的 $\pi$ 共轭部分连接到中心芳环上形成 交叉共轭 $\pi$ 体系的结构, 在中心芳环上引入适当的供电 子基团和吸电子基团赋子了十字架型分子空间上分离 的前线分子轨道(FMOs) ${ }^{[15 \sim 18]}$, 这种独特的空间上分离 的 FMOs 使其最高占有轨道(HOMO) 和最低空轨道
(LUMO)可以相对独立地被溶剂的极性、 $\mathrm{pH}$ 值、可配位 的金属离子等外界环境所影响，从而表现出不同的苂光 性质. 我们在间二咪唑苯的中心苯环上对称地引入两个 强供电子基团，4- $N, N$-二甲基氨基苯乙炔基，构筑了一 种新型的、具有推拉电子效应的、双供体- $\pi$-双受体型的 十字架型分子 $\mathbf{4 a}$, 如图式 1 所示. 为了研究烷基链对分 子的堆积状态和荧光性质的影响, 我们同时合成了 $N, N$ 二丁基氨基取代的分子 $\mathbf{4 b}$. 分子 $\mathbf{4}$ 经过咪坐季铵化反应 得到了分子 5 , 带正电荷的咪唑鎓较咪唑增强的吸电子 能力, 将影响十字架型分子的苂光性质. 研究表明, 含 咪唑的分子 4 具有溶致变色和聚集诱导发光的特性. 同 时, 4 不仅可以在溶液状态下识别 15 种有机酸, 也能在 固体状态下识别 $\mathrm{HCl}$ 气体, 具有较高的实际应用价值. 而含咪唑鎓的分子 $\mathbf{5}$ 不具有聚集诱导发光和识别有机酸 的性质. 烷基链基本未对含咪唑分子 4 的荧光性质产生 影响, 但对含咪唑鎓分子 $\mathbf{5}$ 的固体荧光性质产生了明显 影响.

\section{2 结果与讨论}

\section{1 目标分子的合成}

十字架型荧光分子的合成路线如图式 1 所示.

首先，由间二溴苯与单质碘在强酸条件下反应得到 中心骨架分子 1,5-二溴-2,4-二碘苯 2 . 分子 2 含有两种不 同反应活性的，在空间上呈十字架型排列的四个反应位 点, 为选择性地分步引入供电子基和吸电子基提供了保 障. 供电子基 4- $N, N$-二烷基氨基苯乙炔 $\mathbf{1 a}$ 和 $\mathbf{1 b}$ 分别

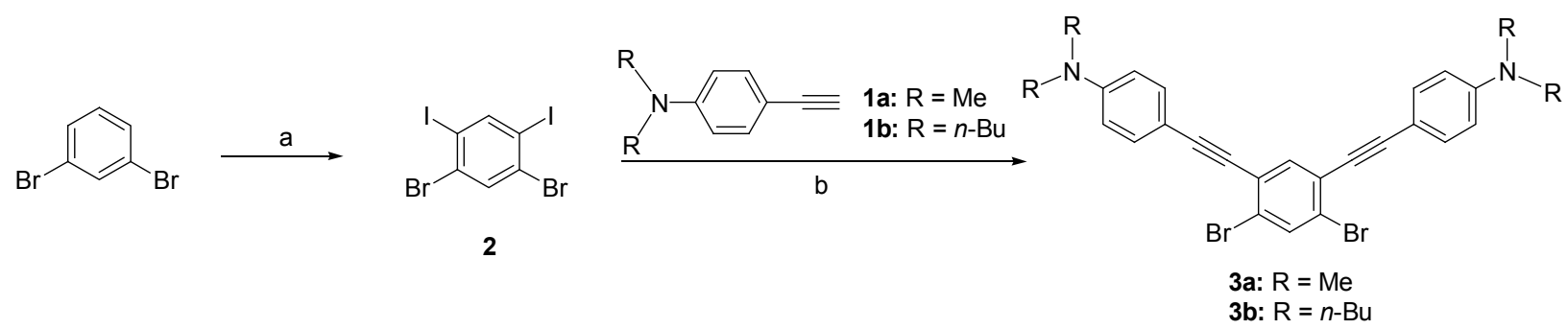

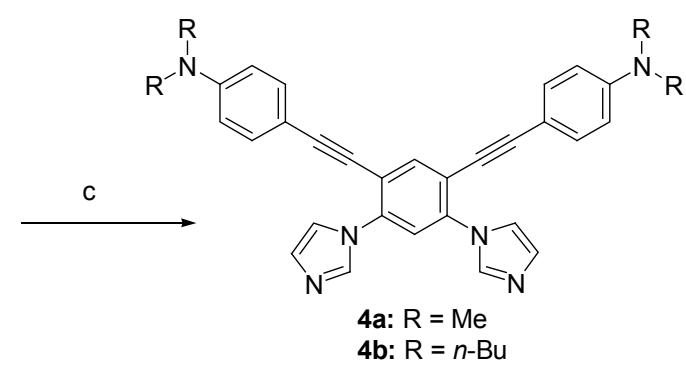

4b: $\mathrm{R}=n-\mathrm{Bu}$

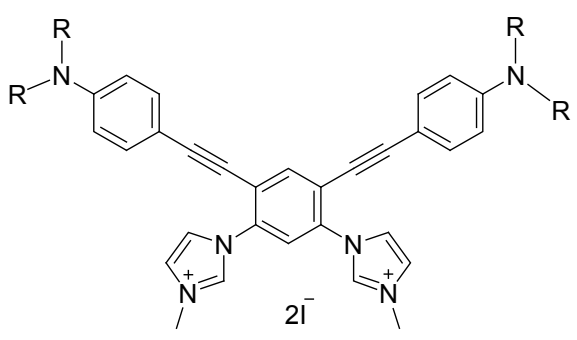

5a: $\mathrm{R}=\mathrm{Me}$

(a) $\mathrm{I}_{2}, \mathrm{H}_{2} \mathrm{SO}_{4}, 125^{\circ} \mathrm{C}, 6 \mathrm{~h}$; (b) $\mathrm{Pd}\left(\mathrm{PPh}_{3}\right)_{2} \mathrm{Cl}_{2}, \mathrm{PPh}_{3}, \mathrm{Cul}, \mathrm{Et}_{3} \mathrm{~N}, \mathrm{THF}, 40{ }^{\circ} \mathrm{C}, 12 \mathrm{~h}$;

(c) N,N-dimethylglycine hydrochloride, imidazole, Cul, $\mathrm{K}_{2} \mathrm{CO}_{3}$, DMF, $120{ }^{\circ} \mathrm{C}, 48 \mathrm{~h}$; (d) Mel, acetonitrile, reflux, $16 \mathrm{~h}$.

图式 1 十字架型分子的合成路线

Scheme 1 Synthesis of cruciforms 4 and 5 
由 $N, N$-二烷基对溴苯胺和三甲基硅基乙炔通过 Sonogashira 反应偶联后，再在碳酸钾存在下脱除三甲 基硅得到. 利用碘比溴高的反应活性, 分子 2 和 4- $N, N-$ 二烷基氨基苯乙炔在 Sonogashira 反应中, 通过控制底 物 4- $N, N$-二烷基氨基苯乙炔的当量和反应温度, 分别以 $56 \%$ 和 $67 \%$ 的产率得到碘与炔偶联的产物 $3 \mathbf{a}$ 和 $3 \mathbf{b}$. 用 $N, N$-二甲基甘氨酸盐酸盐作配体, 碘化亚铜作催化剂, 分子 3 和咪坐经过 $N$-芳基化反应分别以 $56 \%$ 和 45\%的 产率得到间二咪唑十字架型分子 $4 \mathbf{a}$ 和 $\mathbf{4 b}$. 最后, 以碘 甲烷为季铵化试剂, 在乙腈中分别以 $45 \%$ 和 $46 \%$ 的产率 得到间二咪唑鎓十字架型分子 $5 \mathrm{a}$ 和 $5 \mathrm{~b}$. 分子 4 和 5 的 结构经由氢谱、碳谱和高分辨质谱确定.

\section{2 基本光学性质}

十字架型分子 4 和 5 以苯环为中心芳环, 两个处于 间位的咪唑(鎓)为吸电子基团以及两个分别处于它们对 位的 4- $N, N$-二烷基氨基苯乙炔为供电子基团, 形成双供 体- $\pi$-双受体(diDonor- $\pi$-diAcceptor)型苂光分子, 它们的 基本菼光性质见支持信息中 Table S1. 含咪唑的分子 $\mathbf{4 a}$ 是一种黄色的粉末，紫外灯下有黄绿色荧光(图 1a, 1b), 在不同有机溶剂中具有正的溶致变色性质, 其溶液苂光

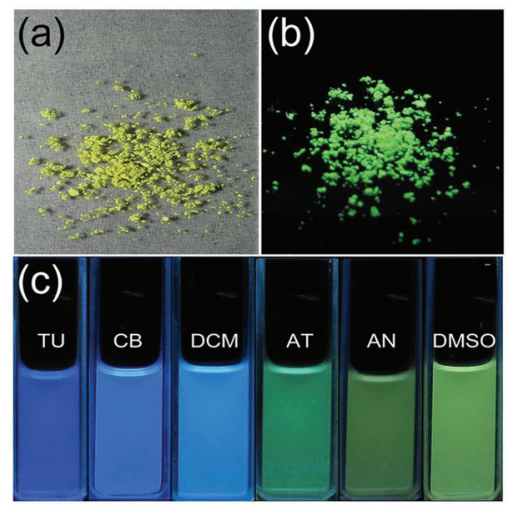

(d)

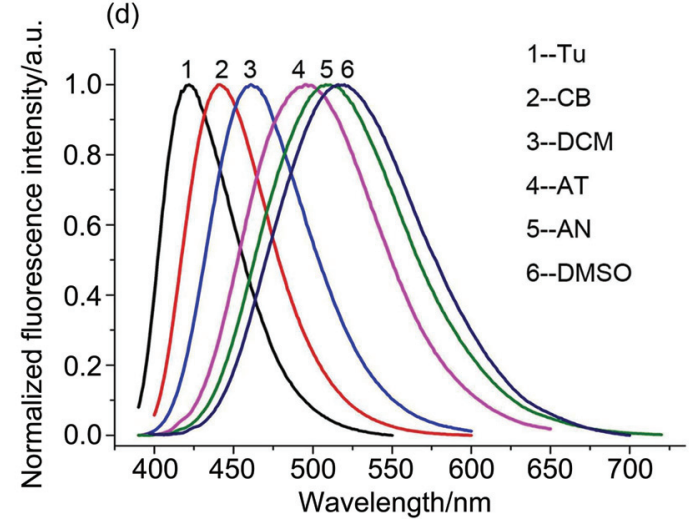

图 1 固态分子 $\mathbf{4 a}$ 在日光下 (a)紫外灯下(b)的照片, 分子 $\mathbf{4 a}$ 在不同溶 剂中的紫外灯下照片(c)和荧光发射谱图(d)

Figure 1 Photographs of $\mathbf{4 a}$ in the solid state under ambient light (a) and UV light (b); Photographs (c) and fluorescence spectra (d) of 4a (1.0 $\left.\times 10^{-6} \mathrm{~mol} / \mathrm{L}\right)$ in different solvents $(\mathrm{TU}=$ toluene, $\mathrm{CB}=$ chlorobenzene, $\mathrm{DCM}=$ dichloromethane, $\mathrm{AT}=$ acetone, $\mathrm{AN}=$ acetonitrile, $\mathrm{DMSO}=$ dimethylsulfoxide) at room temperature
颜色覆盖了由深蓝色到橙黄色的区域(图 1c，1d). 在甲 苯中为深蓝色的荧光 $(422 \mathrm{~nm})$, 在二氯甲烷中为蓝绿色 的苂光 $(461 \mathrm{~nm})$, 在丙酮中为黄绿色的苂光 $(496 \mathrm{~nm})$ 和 在 DMSO 中为橙黄色的荧光 $(516 \mathrm{~nm})$. 随着溶剂极性的 增加, $4 \mathbf{a}$ 的最大发射波长红移, 苂光强度降低. 其在 DMSO 中的苂光波长比其在甲苯中的波长红移了 $94 \mathrm{~nm}$ (Table S2). 这种正的溶致变色性质是具有分子内电荷 转移(Intramolecular Charge Transfer, ICT)效应的 D- $\pi-A$ 型苂光分子的典型特征. ICT 效应部分是由于极性溶剂 相对于非极性溶剂更能稳定电荷转移激发态，而其基态 相对的不受溶剂极性的影响. 因此, 极性溶剂能够显著 的减小能带间隙，导致荧光波长在极性溶剂中比在非极 性溶剂中要红移. 在乙腈中分子 $4 \mathrm{a}$ 的斯托克斯位移高 达 $148 \mathrm{~nm}$, 表明其激发态与基态结构有较大变化. 大的 斯托克斯位移可以避免激发光和散射光的干扰和由于 自吸收导致的荧光强度降低，从而提高荧光分子的响应 灵敏度.

含咪唑鎓的分子 $5 \mathbf{a}$ 是一种红色的粉末，紫外灯下 发出红色荧光(图 $2 a, 2 b$ ), 也具有明显的正的溶致变色 性质. 在不同极性溶剂中, 分子 $\mathbf{5 a}$ 的荧光颜色覆盖了由 蓝色到红色的区域(图 2c, 2d). 在 THF 中发射蓝色的荧 光(444 nm), 在氯仿中发射绿色的苂光(519 nm), 在丙
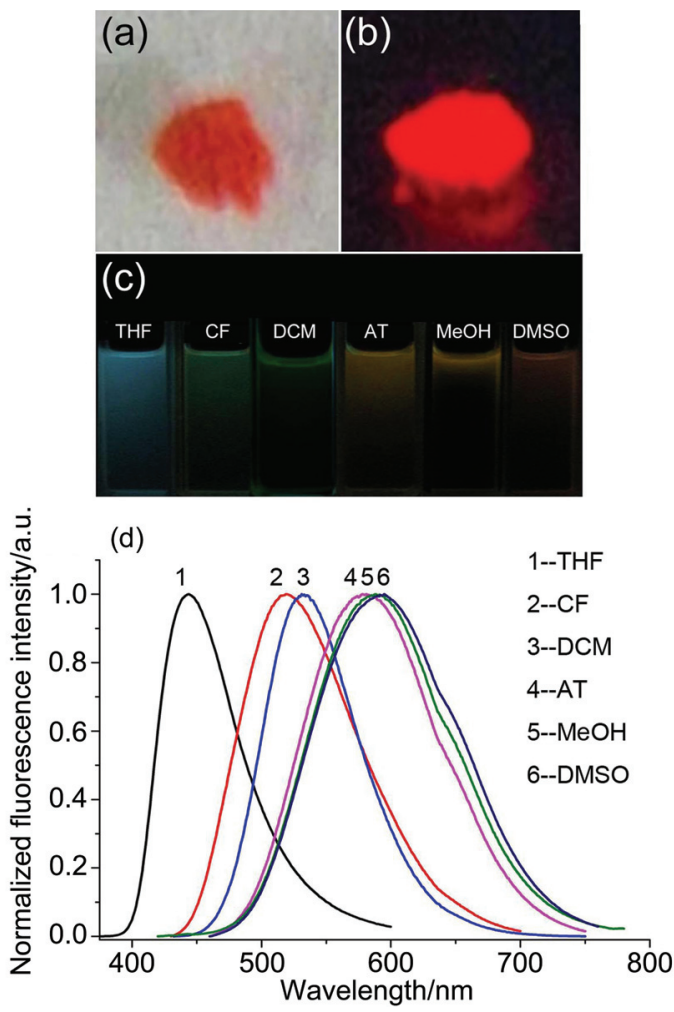

图 2 固态分子 $\mathbf{5 a}$ 在日光下 (a)紫外灯下 $(b)$ 的照片, 分子 $\mathbf{5 a}$ 在不同溶 剂中的紫外灯下照片(c)和荧光发射谱图(d)

Figure 2 Photographs of 5a in the solid state under ambient light (a) and UV light (b); Photographs (c) and fluorescence spectra (d) of 5a (1.0 $\left.\times 10^{-6} \mathrm{~mol} / \mathrm{L}\right)$ in different solvents $(\mathrm{CF}=$ chloroform $)$ at room temperature 
酮中发射橙黄色的荧光(574 nm), 在 DMSO 中发射红色 的苂光 $(595 \mathrm{~nm})$, 其在 DMSO 中的荧光波长比其在 THF 中的波长要红移 $151 \mathrm{~nm}$. 随着溶剂极性的增加, 5a 的最 大发射波长红移，苂光强度显著降低. 与分子 $\mathbf{4 a}$ 相比, 分子 $\mathbf{5 a}$ 在溶液中的苂光波长红移, 量子产率降低, 并且 $5 \mathbf{a}$ 的溶致变色性质更加明显. 溶液极性对分子的荧光 性质影响显著, 是潜在的环境极性传感器 ${ }^{[19]}$.

从表 $\mathrm{S} 1$ 可以看出: 在溶液状态下, 不论是季铵化 之前的分子 $4 \mathrm{a}$ 和 $4 \mathrm{~b}$, 还是季铵化之后的分子 $5 \mathrm{a}$ 和 $\mathbf{5 b}$, 带甲基的化合物和带丁基的化合物的菼光性质都很类 似，这说明 $\mathrm{R}$ 基团对分子的溶液荧光性质的影响不大. 甲基和丁基是很弱的供电子基，相对于苯胺基团，它们 对分子的电子云分布的影响不大, 对分子的跃迁能级的 影响不大，因此对分子的溶液苂光性质影响不大. 四个 分子都是推拉电子体系, 因此都具有溶致变色性质. 季 铵化之后, 由于电子受体咪唑鎓的吸电子能力要远强于 咪坐, 分子内的推拉电子效应更加明显, ICT 效应增强, 因此分子的紫外吸收波长和荧光波长都相对红移, 量子 产率降低.

在固体状态下, 分子 $\mathbf{4 a}$ 发射较强的黄绿色荧光, 苂 光量子产率为 0.33 . 分子 $\mathbf{4 a}$ 中的两个咪唑基团直接连 接到中心苯环上, 虽然处于间位, 但仍然在空间上存在 一定的拥挤, 从而导致咪唑基团与中心苯环并非共平 面, 而是具有一定的夹角. 这种扭曲的构型降低了分子 间 $\pi-\pi$ 堆积相互作用程度, 减小了分子间 $\pi$ 轨道的耦合 导致的激发态能量损失, 因而分子 $4 \mathbf{a}$ 具有较强的固体 荧光 ${ }^{[20]}$. 分子 $5 \mathbf{a}$ 在固体状态下发射红色荧光, 波长为 $632 \mathrm{~nm}$, 荧光量子产率为 0.14 . 其色度坐标为 $(x=0.67$, $y=0.32)$, 与国际照明委员会(Commission Internationale de L'Eclairage, CIE)确立的标准红色坐标 $(x=0.67, y=$ $0.33)$ 吻合的非常好. 目前, 5a 是首例具有红色固态荧光 的咪唑鎓骨架苂光分子, 有望在红色发光器件中得到应 用.

烷基链对十字架型分子 $\mathbf{4}$ 和 $\mathbf{5}$ 在溶液状态下的荧光 性质几乎没有影响, 但在固体状态下, 季铵化之前的分 子 $4 \mathrm{a}$ 和 $4 \mathrm{~b}$ 的苂光性质类似, 而季铵化之后的分子 $\mathbf{5 a}$ 和 $5 \mathbf{b}$ 的荧光波长却相差 $106 \mathrm{~nm}$ (Table S1). 这表明在固 体状态下, 烷基链对间二咪唑十字架分子 4 的堆积模式 影响不大，却对间二咪唑鎓十字架分子 5 的堆积模式影 响很大, 从而影响了分子的固态苂光性质. 为了解释这 一现象, 我们分别制备了质量分数为 $0.1 \%$ 的 $5 \mathbf{a}$ 和 $5 \mathbf{b}$ 的 聚甲基丙烯酸甲酯(PMMA)薄膜, 测试了它们的苂光性 质. 我们发现二者在薄膜中的苂光波长和量子产率都是 类似的, 这与二者在溶液中的苂光性质类似是吻合的. 同时，两者的 PMMA 薄膜的荧光波长(5a: $484 \mathrm{~nm}, \mathbf{5 b}$ : $485 \mathrm{~nm}$ )相对于固体状态都要蓝移, 薄膜荧光量子产率 (5a: 0.44, 5b: 0.47)也近似, 并都高于固体苂光量子产率. 在 PMMA 薄膜状态下, 分子之间不存在堆积, 不存在分
子间相互作用，因此分子 $\mathbf{5 a}$ 和 $\mathbf{5 b}$ 的薄膜苂光性质类似. 但在固体状态下，二者可能存在不同的堆积模式，具有 不同程度的分子间作用力，从而导致荧光波长发生不同 程度的红移，量子产率降低.

\section{3 聚集诱导发光的性质}

传统的荧光分子在稀溶液中具有高的荧光量子产 率，但是在浓溶液或固体状态下荧光减弱甚至荧光发生 淬灭, 即聚集诱导荧光淬灭 (Aggregation-caused Quenching, ACQ). ACQ 效应不利于荧光材料在固体光 电学器件中的应用. 2001 年, Tang 课题组观察到一个很 有趣的现象: 硅杂环成二烯衍生物在稀溶液中几乎没有 荧光，但聚集态的荧光强度大大增加. 他们将此现象称 为聚集诱导发光(Aggregation-induced Emission, AIE)现 象 ${ }^{[21]}$. AIE 能够克服荧光分子聚集诱导荧光淬灭的难题, 受到了广泛的关注和研究, 目前, 已有越来越多结构各 异的 AIE 分子被设计开发出来.

Tang 认为, 如果一个分子的 $\pi$ 共轭中心连有多个旋 转受限的芳(杂)环基团, 就可能会产生分子内旋转受阻 (Restricted Intramolecular Rotation, RIR)效应，这个分子 就有可能具有 $\mathrm{AIE}$ 性质. 分子 $\mathbf{4 a}$ 中咪唑基团和 $N, N$-二 甲基苯胺基团与 $\pi$ 共轭中心以单键相连接, 苯环与咪唑 环的旋转可导致分子共轭程度的变化, 进而影响荧光强 弱. 因此, 分子 $\mathbf{4 a}$ 可能具有 AIE 的性质.

我们在分子 $4 \mathbf{a}$ 的甲醇溶液 $\left(10^{-4} \mathrm{~mol} / \mathrm{L}\right)$ 中分别加入 不同比例的水，配置了水的体积分数分别为 $0 \% \sim 90 \%$ 的分子 $4 \mathbf{a}$ 的混合溶液 $\left(10^{-5} \mathrm{~mol} / \mathrm{L}\right)$, 测得了它们在相同 条件下的苂光发射强度和苂光量子产率，如图 3 所示. 当混合溶剂中水的含量小于 $50 \%$ 时，溶液的苂光强度和 苂光量子产率都随水的含量增加而递减，并且苂光波长 向长波方向移动. 此现象可解释为: 水的含量小于 $50 \%$ 时, 分子 $4 \mathbf{a}$ 还没有聚集. 此时水的含量增加, 溶剂的极 性增大，分子的荧光波长红移，苂光强度降低，这是典 型的 ICT 效应. 当水的含量为 $60 \%$ 和 $70 \%$ 时，分子的苂 光强度和苂光量子产率显著增加, 此时分子 $4 \mathbf{a}$ 已经形 成聚集态. 当水含量到达 $70 \%$ 时, 苂光强度相对于纯甲 醇溶液提高了 4 倍，相对于水含量 $50 \%$ 的溶液提高了 12 倍多; 苂光量子产率相对于纯甲醇溶液提高了 8 倍, 相 对于水含量 $50 \%$ 的溶液提高了 28 倍多. 在稀溶液状态 下, 分子 $4 \mathbf{a}$ 中碳碳单键的自由旋转消耗了分子激发态 能量, 非辐射衰变通道打开, 荧光淬灭. 随着溶液中水 含量的增加, 分子 $\mathbf{4 a}$ 形成聚集态, 其分子内碳碳单键的 旋转受阻，导致非辐射衰变通道被抑制，辐射跃迁增强， 从而荧光增强, 表现出 AIE 性质 ${ }^{[22,23]}$. 而当水的含量为 $80 \%$ 和 $90 \%$ 时，分子的苂光强度和苂光量子产率略有下 降. 其可能的原因是分子 $\mathbf{4 a}$ 在溶液中的聚集形态发生 了变化.

在甲醇/水的混合溶剂中, 分子 $\mathbf{4 b}$ 的荧光强度随着 水含量的变化与分子 $\mathbf{4 a}$ 呈现类似的改变(Figure S2). 而 


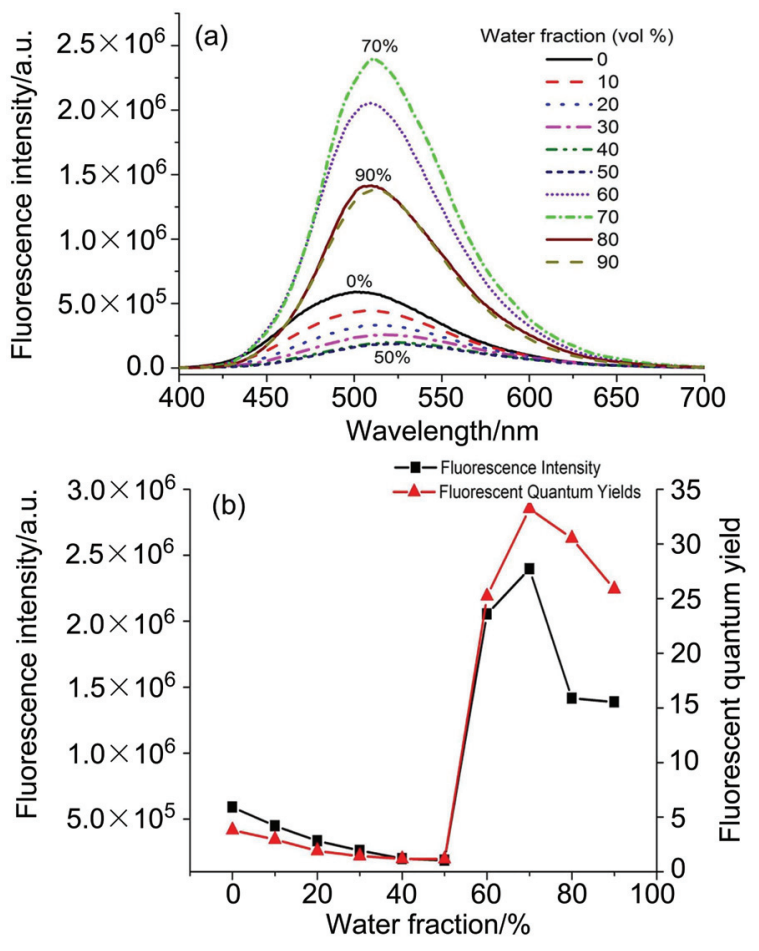

图 3 (a) 分子 $4 \mathrm{a}$ 在甲醇和水的混合溶剂 $\left(1.0 \times 10^{-5} \mathrm{~mol} / \mathrm{L}\right)$ 中的荧光光 谱; (b)苂光发射强度和苂光量子产率随水含量变化的相关图

Figure 3 (a) Fluorescence spectra of $\mathbf{4 a}$ in water/methanol mixtures (1.0 $\times 10^{-5} \mathrm{~mol} / \mathrm{L}$ ), (excited at $370 \mathrm{~nm}$ ); (b) Changes in the fluorescence intensity and quantum yield of $\mathbf{4 a}$ with different water fractions

季铵化后的分子 $5 \mathrm{a}$ 和 $\mathbf{5 b}$ 在大极性溶剂(如：丙酮、乙腈、 甲醇等)中的溶解性很好, 加入不良溶剂(如: 水、乙醚、 正己烷等)也没有形成聚集态. 因此, $\mathbf{5 a}$ 和 $\mathbf{5 b}$ 不具有 AIE 性质.

\section{4 对有机酸的识别}

羧酸及其衍生物是一类非常重要的有机物, 广泛存 在于生物体、药物分子和生产原料及中间体中. 美国亚 利桑那大学统计的前 200 名知名品牌的药物中有 23 种 药物含有羧基基团 ${ }^{[24]}$. 因此, 定性的鉴别有机酸, 特别 是那些结构类似的有机酸, 对于鉴定假冒的、过期的药 物具有实际应用价值. 分子 $\mathbf{4 a}$ 的供电子基团 4- $N, N-$ 二 甲氨基苯乙炔和吸电子基团咪唑都含有带孤对电子的 氮原子, 四个氮原子的电子云密度和所处的环境不同导 致它们与不同的有机酸作用的强弱有所区别, 因此分子 $4 \mathbf{a}$ 的 HOMO 和 LUMO 可以被具有不同酸性和结构的有 机酸相对独立的影响, 从而导致分子的荧光性质发生不 同的变化, 达到识别有机酸的目的. 最近, 也有文献报 道利用咪唑和羒酸的相互作用来实现手性药物的识 别 ${ }^{[25]}$. 为了简化识别程序, 我们选择用数码照相的形式 记录荧光颜色，代替复杂繁琐的苂光光谱仪 ${ }^{[26]}$.

本文识别的有机酸 $\mathrm{C} 1 \sim \mathrm{C} 15$ 有类似的结构和近似 的 $\mathrm{p} K_{\mathrm{a}}$ 值(Figure $\mathrm{S} 3$ ). 每种有机酸都分别溶解在二氯甲 烷和乙腈中, 浓度大约为 $1 \mathrm{~g} / \mathrm{L}$. 主体分子溶解在二氯甲
烷溶液中, 浓度为 $1.0 \times 10^{-4} \mathrm{~mol} / \mathrm{L} .20 \mu \mathrm{L}$ 的主体分子溶 液分别与 $2.0 \mathrm{~mL}$ 有机酸的溶液混合, 得到的混合溶液 中有机酸与分子 $4 \mathrm{a}$ 的摩尔分子比为 $7.2 \times 10^{3}: 1$. 将装 有混合溶液的样品池置于紫外灯下, 用 $365 \mathrm{~nm}$ 的紫外 灯激发. 样品的苂光颜色用数码相机记录, 为了避免样 品荧光之间的干扰，每次只拍摄一个样品. 一共有 16 种 样品(包括不加酸的分子 $\mathbf{4 a}$ )和 2 种溶剂, 将得到的 32 张 照片用 PhotoShop 软件编辑, 分别裁剪出代表每种溶液 的荧光颜色的，大小相等的正方形小块，将这些正方形 小块按一定顺序排列, 即得到了如图 4 所示的识别阵列.

从图 4 中我们可以观察到在二氯甲烷和乙腈中 15 种有机酸可以很好的区分开, 分子 $\mathbf{4 a}$ 和 15 种有机酸在 两种溶剂中显示了不同的苂光颜色. 一些结构类似的有 机酸可以很好的区分开. 有机酸 $\mathrm{C} 1, \mathrm{C} 2$ 和 $\mathrm{C} 3$ 是苯甲酸 衍生物; $\mathrm{C} 4, \mathrm{C} 5$ 和 $\mathrm{C} 6$ 都是杂环有机酸; C7 $\sim \mathrm{C} 14$ 都是结 构类似的脂肪族多取代羧酸: 它们之间要么碳链只相差 一个碳原子, 要么互为顺反异构, 要么带有不同的取代 基. 选取的大多数脂肪族有机酸都存在于生物体内, 并 且对维持生物体的生命是非常重要的. 其中, 有机酸 C4 俗称烟酸或维生素 B3, 在人体内有着很重要的生理作 用. 同时, 一些具有相近 $\mathrm{p} K_{\mathrm{a}}$ 值的有机酸也能很好的相 互区别. 比如: 有机酸 $\mathrm{C} 1, \mathrm{C} 7, \mathrm{C} 10, \mathrm{C} 11$ 和 $\mathrm{C} 14$ 的 $\mathrm{p} K_{\mathrm{a}}$ 值大约为 $3.0, \mathrm{C} 2, \mathrm{C} 3$ 和 $\mathrm{C} 12$ 的 $\mathrm{p} K_{\mathrm{a}}$ 值大约为 3.4. 大多 数有机酸都导致分子 $\mathbf{4 a}$ 的苂光波长红移, 特别是在二 氯甲烷中. 荧光波长的红移有可能是因为分子 $4 \mathrm{a}$ 的咪 唑基团优先被质子化，使得分子的推拉电子效应更加明 显，导致荧光波长红移.

我们利用 Adobe PhotoShop 软件将荧光的颜色进行 量化处理. 每个分析物在两种溶剂中都有各自的 R(ed), G(reen)和 B(lue)数值, 这样每个分析物就分别带有 6 个 数值. 我们首先将目光集中到 15 种有机酸中的任意两 种. 利用公式 Eq. 1 (Supporting Information), 我们得到 了每个有机酸在两种溶剂中与另一种有机酸之间的相 对标准方差 $\sigma$, 其值代表着每种有机酸和另一种有机酸 分别与分子 $4 \mathrm{a}$ 混合最后得到的溶液荧光颜色的相对差 异大小. 有机酸与相对标准方差之间作图可以得到三维 柱状图(图 5), 柱状图说明用这种方法可以很好的区分 这 15 种有机酸. 例如: $\mathrm{C} 9$ 和 $\mathrm{C} 10$ 互为顺反异构体, 在柱 状图中分别以 $\mathrm{C} 9$ 和 $\mathrm{C} 10$ 坐标作垂线交于一点, 此点上 的长方体为蓝色, 通过 $\mathrm{Z}$ 轴可以得知其标准方差值为 112 , 处于高水平, 说明 $\mathrm{C} 9$ 和 $\mathrm{C} 10$ 的区分度非常好. $\mathrm{C} 2$ 和 $\mathrm{C} 3$ 的 $\mathrm{p} K_{\mathrm{a}}$ 值只差 0.01 , 用相同的方法读取其标准方差 为 117 , 区分度也非常好. $\mathrm{C} 1$ 和 $\mathrm{C} 2$ 具有类似的结构, 以 它们的坐标作垂线交于长方体为深红色，其值为 68 , 区 分度较好. C5 和 C6 的结构高度相似, 它们的长方体为 灰色, 标准方差值为 14 , 仍然能够区分开来.

随后，利用公式 Eq. 2 (Supporting Information)我们 得到了每个分析物分别在二氯甲烷和乙腈两种溶剂中 


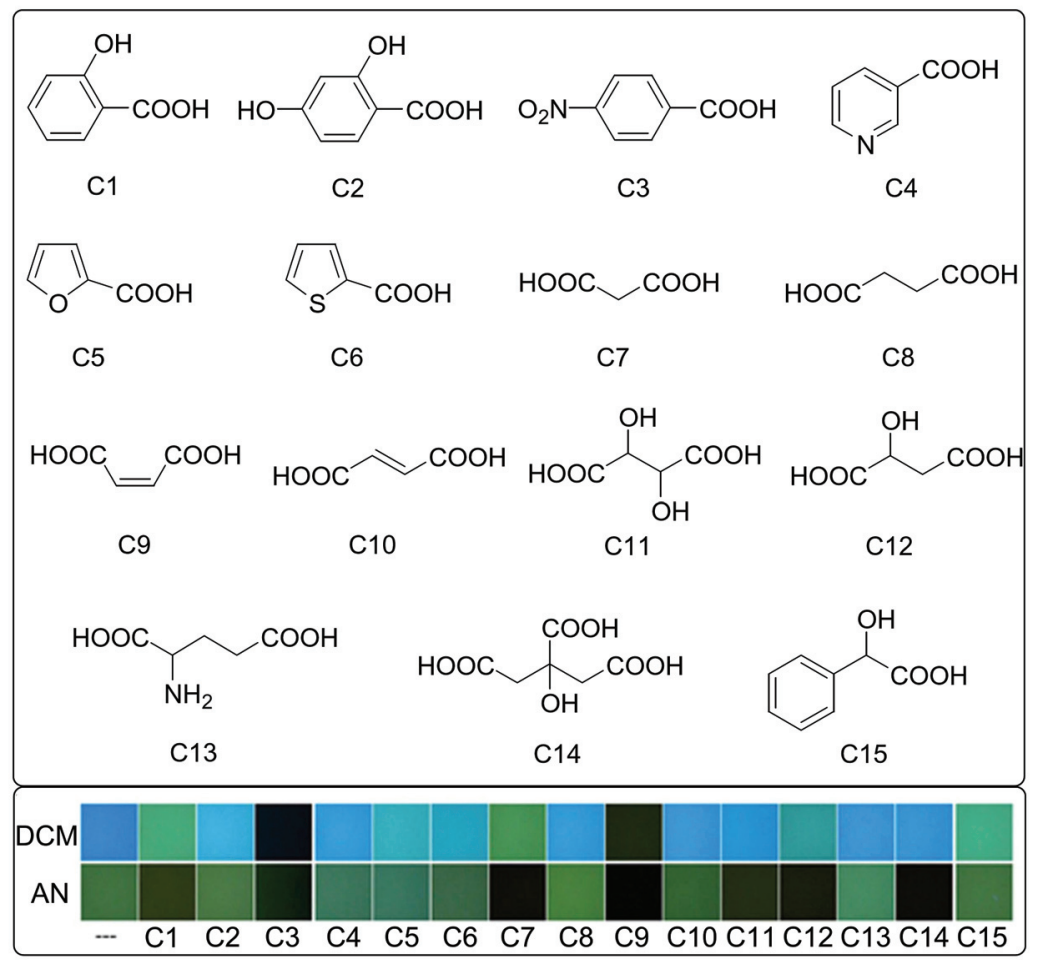

图 4 上图为本文分析的有机酸的结构式, 下图为分子 $4 \mathrm{a}$ 分别在二氯甲烷和乙腈中与有机酸混合后的溶液的照片 $(365 \mathrm{~nm}$ 激发)

Figure 4 The structures of the carboxylic acids and the emission colors of 4a upon mixed with them in DCM and AN (excited at $365 \mathrm{~nm}$ )

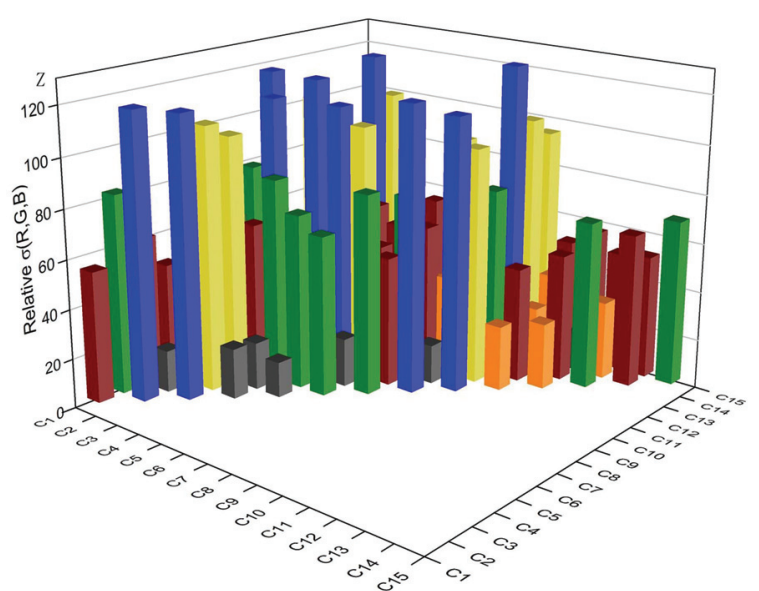

图 5 有机酸 $\mathrm{C} 1 \sim \mathrm{C} 15$ 中任意两种酸的 $\mathrm{R} / \mathrm{G} / \mathrm{B}$ 值之间的标准方差的相 关图

Figure 5 The correlation diagram of standard deviations of R/G/B values of analytes $\mathrm{C} 1 \sim \mathrm{C} 15$ relative to all other analytes

的 $\mathrm{R} / \mathrm{G} / \mathrm{B}$ 值与分子 $\mathbf{4 a}$ 的空白溶液之间的标准方差 $\sigma^{\prime}$, 其 值代表着每种分析物与分子 $\mathbf{4 a}$ 混合后，分子 $\mathbf{4 a}$ 的苂光 颜色改变的程度. 我们得到了两种溶液中有机酸 $\mathrm{p} K_{\mathrm{a}}$ 值 与相应的标准方差的相关图(Figure S4). 在二氯甲烷中, 有机酸的酸性和苂光颜色改变的程度之间没有统计学 上的相关性. 在乙腈中, 部分有机酸的 $\mathrm{p} K_{\mathrm{a}}$ 值越小, 即 酸性越强, 得到的 $\sigma^{\prime}$ 值也越大, 分子 $\mathbf{4 a}$ 的苂光颜色改变 越大. 这可能是由于有机酸的酸性越强, 有机酸与分子 4a 之间的相互作用也越强, 从而导致荧光颜色改变越 明显.
含长链丁基的二咪唑十字架型分子 $4 \mathrm{~b}$ 和含甲基的 分子 $4 \mathrm{a}$ 具有相同的识别能力. 但是, 咪唑季铵化后的十 字架型分子 $\mathbf{5}$ 与有机酸接触后, 其溶液荧光颜色基本上 没有改变，因而没有识别的作用. 季铵化后的分子 $\mathbf{5}$ 只 剩下两个 $N, N$-二烷基苯胺中的带孤对电子的氮原子，同 时咪唑鎓的强吸电子效应，使得这两个氮原子上的电子 云密度降低，与有机酸的相互作用减弱，无法引起分子 荧光性质的改变. 因此, 咪唑上 3 位氮原子以及咪唑适 当的吸电子能力对于识别有机酸起着至关重要的作用.

\section{5 固体传感器-对 $\mathrm{HCl}$ 气体的识别}

在溶液中，分子 4 实现了对 15 种有机酸的识别. 同 时, 由于其具有较强的固态荧光, 因此也有可能在固体 状态下对酸性气体进行识别 ${ }^{[27]}$. 我们将硅胶板置于分 子 $4 \mathrm{a}$ 的二氯甲烷溶液中数分钟, 取出烘干制得识别板. 将识别板置于玻璃器血中, 向其中通入 $\mathrm{HCl}$ 气体, 其苂 光颜色由黄绿色变为蓝色. 随后, 将识别板置于三乙胺 的氛围中, 其苂光颜色又恢复到初始状态(图 6). 这个简 单的酸碱识别实验说明分子 4 有潜力作为固体传感器实 现对酸性气体的识别.

虽然分子 $\mathbf{5}$ 在溶液中无法区分有机酸, 但其固体却 同分子 4 一样具有识别 $\mathrm{HCl}$ 气体的能力. 例如, 分子 $\mathbf{5 a}$ 在 $\mathrm{HCl}$ 和 $\mathrm{Et}_{3} \mathrm{~N}$ 气体的作用下, 其荧光颜色也能发生可 逆的变化(图 7). 因此分子 $\mathbf{5}$ 也能作为固体传感器用于 识别 $\mathrm{HCl}$ 气体. 


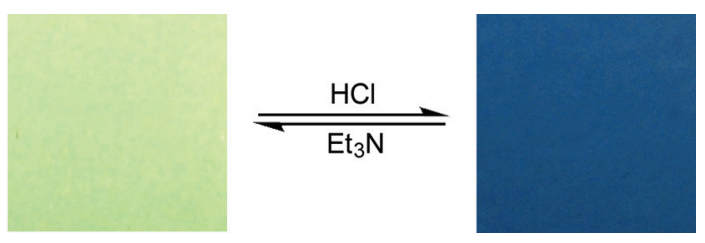

图 6 分子 $4 \mathrm{a}$ 的硅胶板接触酸和碱后紫外灯下的照片

Figure 6 The pictures of silica plate coated with 4a under UV light upon exposure to $\mathrm{HCl}$ and $\mathrm{Et}_{3} \mathrm{~N}$

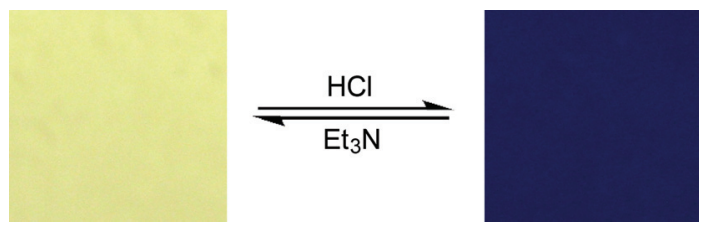

图 7 分子 $5 \mathrm{a}$ 的硅胶板接触酸和碱后紫外灯下的照片

Figure 7 The pictures of silica plate coated with 5a under UV light upon exposure to $\mathrm{HCl}$ and $\mathrm{Et}_{3} \mathrm{~N}$

\section{3 结论}

通过 Sonogashira 偶联反应、 $N$-芳基化反应和季铵 化反应，我们构筑了一类新型的基于咪唑(鍮)的十字架 型苂光分子. 分子 $4 \mathrm{a}$ 具有正的溶致变色效应, 在乙腈溶 液斯托克斯位移高达 $148 \mathrm{~nm}$. 同时, 其具有明显的 AIE 性质和较强的固体苂光. 仅使用分子 $\mathbf{4 a}$ 在二氯甲烷和 乙腈两种溶剂中, 通过数码照相的方式, 15 种结构类似 或 $\mathrm{p} K_{\mathrm{a}}$ 相近的有机酸成功地被区分开来, 相较于传统使 用荧光仪的方法, 该方法更为简单直观. 将荧光颜色转 化为 $\mathrm{R} / \mathrm{G} / \mathrm{B}$ 数值, 通过计算标准方差, 从统计学的角度 实现了分子 4a 对这 15 种有机酸的量化识别. 另外, 烷 基链对间二咪唑十字架型分子 $\mathbf{4}$ 的荧光性质没有明显影 响, 但却显著影响了间二咪唑鎓十字架型分子 $\mathbf{5}$ 在固态 下的荧光性质, $\mathbf{5}$ 是一个具有高色纯度的红色固体荧光 材料. 最后, 分子 4 和 5 均表现出固态识别 $\mathrm{HCl}$ 气体的 能力. 间二咪唑十字架型分子 4 有望作为新型的荧光材 料应用于识别区分药物分子领域, 间二咪唑鎓十字架型 分子 $5 \mathbf{a}$ 有望应用于红色发光器件, 展现了间二咪唑(鎓) 苯在构筑有机功能性材料方面的新应用.

\section{4 实验部分}

\section{1 目标化合物的合成与表征}

目标化合物的合成路线见图式 $1,4-N, N-$ 二烷基氨 基苯乙炔 $1 \mathbf{a}$ 和 $\mathbf{1 b}$ 和 1,5-二溴-2,4-二碘苯 2 参照文献[28, 29]的方法制备, 化合物 3a, 4a 和 5a 的具体合成方法如 下.

4.1.1 4,6-二溴-1,3-二(4- $N, N$-二甲基氨基)苯乙炔基苯 (3a)的合成

将原料 1,5-二澳-2,4-二碘苯 $731.6 \mathrm{mg}(1.50 \mathrm{mmol})$ 、 $4-N, N$-二甲氨基苯乙炔 $468.3 \mathrm{mg}(3.20 \mathrm{mmol})$ 、二(三苯 基膦)二氯化钯 $31.6 \mathrm{mg}(0.05 \mathrm{mmol})$ 、三苯基膦 $19.7 \mathrm{mg}$
$(0.08 \mathrm{mmol})$ 和碘化亚铜 $14.3 \mathrm{mg}(0.08 \mathrm{mmol})$ 加入到支管 中, 氮气置换三次, 充入氮气后向体系中注入 $5 \mathrm{~mL}$ 三 乙胺和 $5 \mathrm{~mL}$ 四氢呋喃. 体系置于 $40{ }^{\circ} \mathrm{C}$ 中反应 $12 \mathrm{~h}$. 反 应产生大量固体，用二氯甲烷稀释，抽滤，滤液浓缩， 柱层析分离[展开剂: $V$ (石油醚) : $V$ (乙酸乙酯 $)=5: 1$ ], 得到黄色的固体产物 $440 \mathrm{mg}$, 产率 $56.2 \%$, m.p. 170 $172{ }^{\circ} \mathrm{C} .{ }^{1} \mathrm{H}$ NMR (400 MHz, $\mathrm{CDCl}_{3}$ ) $\delta: 3.01$ (s, 12H), 6.66 (d, $J=8.4 \mathrm{~Hz}, 4 \mathrm{H}), 7.43(\mathrm{~d}, J=8.4 \mathrm{~Hz}, 4 \mathrm{H}), 7.65(\mathrm{~s}, 1 \mathrm{H})$, $7.83(\mathrm{~s}, 1 \mathrm{H}) ;{ }^{13} \mathrm{C}$ NMR $\left(100 \mathrm{MHz}, \mathrm{CDCl}_{3}\right) \delta: 40.3,85.3$, $97.0,109.2,111.8,123.8,125.6,133.0,135.4,135.7$, 150.6; HRMS $\left(\right.$ ESI $^{+}$): calcd for $\mathrm{C}_{26} \mathrm{H}_{23} \mathrm{Br}_{2} \mathrm{~N}_{2}[\mathrm{M}+\mathrm{H}]^{+}$ 521.0223, found 521.0230.

4.1.2 4,6-二咪唑-1,3-二(4- $N, N$-二甲基氨基)苯乙炔基 苯 $(4 a)$ 的合成

将原料 4,6-二澳-1,3-二(4- $N, N$-二甲基氨基)苯乙炔 基苯 $391.7 \mathrm{mg}(0.75 \mathrm{mmol}) 、$ 咪唑 $255.3 \mathrm{mg}(3.75 \mathrm{mmol})$ 、 碳酸钾 $621.9 \mathrm{mg}(4.50 \mathrm{mmol}), \mathrm{N}, \mathrm{N}$-二甲基甘氨酸盐酸盐 $41.9 \mathrm{mg}(0.30 \mathrm{mmol})$ 和碘化亚铜 $28.6 \mathrm{mg}(0.15 \mathrm{mmol})$ 加 入到支管中, 氮气置换三次, 充入氮气后向体系中注入 $8 \mathrm{~mL}$ DMF. 体系置于 $120{ }^{\circ} \mathrm{C}$ 中反应 $48 \mathrm{~h}$. 反应结束后 用二氯甲烷稀释, 抽滤, 滤液浓缩, 柱层析分离[展开 剂: $V$ (石油醚) $: V$ (丙酮 $)=1: 1$, 后换用 $V$ (二氯甲烷 $)$ : $V$ (甲醇 $)=30: 1$ ], 得到黄绿色的固体产物 $210 \mathrm{mg}$, 产率 $56.4 \%$, m.p. $158 \sim 160{ }^{\circ} \mathrm{C}$. ${ }^{1} \mathrm{H}$ NMR $\left(400 \mathrm{MHz}, \mathrm{CDCl}_{3}\right) \delta$ : $3.00(\mathrm{~s}, 12 \mathrm{H}), 6.61(\mathrm{~d}, J=8.8 \mathrm{~Hz}, 4 \mathrm{H}), 7.25$ (s, 2H), 7.27 (s, 1H), 7.29 (d, $J=8.8 \mathrm{~Hz}, 4 \mathrm{H}), 7.39$ (s, 2H), 7.87 (s, 1H), 8.01 (s, $2 \mathrm{H}) ;{ }^{13} \mathrm{C}$ NMR $\left(100 \mathrm{MHz}, \mathrm{CDCl}_{3}\right) \delta: 40.1,82.4$, $98.4,108.2,111.7,119.5,120.0,121.1,129.5,132.9$, 136.6, 136.9, 137.3, 150.7; HRMS $\left(\mathrm{ESI}^{+}\right)$: calcd for $\mathrm{C}_{32} \mathrm{H}_{29} \mathrm{~N}_{6}[\mathrm{M}+\mathrm{H}]^{+}$497.2448, found 497.2453. Anal. calcd for $\mathrm{C}_{32} \mathrm{H}_{28} \mathrm{~N}_{6} \cdot 0.25 \mathrm{H}_{2} \mathrm{O}$ : C 76.70 , H 5.73, N 16.77; found C 76.76, H 5.83, N 16.57.

4.1.3 4,6-二咪唑鎓-1,3-二(4- $N, N$-二甲基氨基)苯乙炔 基苯 $(\mathbf{5 a})$ 的合成

将原料 4,6-二咪唑-1,3-二(4- $N, N$-二甲基氨基)苯乙 炔基苯 $92.7 \mathrm{mg}(0.2 \mathrm{mmol})$ 加入到支管中, 氮气置换三 次, 充入氮气后向体系中注入 $0.2 \mathrm{~mL}$ 碘甲烷 $(3.2 \mathrm{mmol})$ 和 $50 \mathrm{~mL}$ 乙腈. 体系置于 $80{ }^{\circ} \mathrm{C}$ 中反应 $16 \mathrm{~h}$, 减压蒸除 乙腈, 柱层析分离 [展开剂: $V$ (二氯甲烷) : $V$ (甲醇 $)=$ $10: 1$ ], 得到红色的固体产物 $70 \mathrm{mg}$, 产率 $45.0 \%$. ${ }^{1} \mathrm{H}$ NMR (400 MHz, DMSO) $\delta: 3.00(\mathrm{~s}, 12 \mathrm{H}), 4.06(\mathrm{~s}, 6 \mathrm{H})$, $6.74(\mathrm{~d}, J=8.4 \mathrm{~Hz}, 4 \mathrm{H}), 7.32(\mathrm{~d}, J=8.0 \mathrm{~Hz}, 4 \mathrm{H}), 8.07$ (s, $2 \mathrm{H}), 8.19(\mathrm{~s}, 1 \mathrm{H}), 8.21(\mathrm{~s}, 1 \mathrm{H}), 8.28(\mathrm{~s}, 2 \mathrm{H}), 9.81(\mathrm{~s}, 2 \mathrm{H})$; ${ }^{13} \mathrm{C}$ NMR (100 MHz, DMSO) $\delta: 36.5,39.6,81.0,100.9$, $106.0,111.8,121.5,123.4,124.1,124.3,133.1,135.6$, 137.7, 151.0; HRMS $\left(\mathrm{ESI}^{+}\right)$: calcd for $\mathrm{C}_{34} \mathrm{H}_{35} \mathrm{I}_{2} \mathrm{~N}_{6}[\mathrm{M}+$ $\mathrm{H}]^{+}$781.1007, found 781.1010 . 


\section{References}

[1] Pugh, D.; Danopoulos, A. A. Coord. Chem. Rev. 2007, 251, 610.

[2] Poyatos, M.; Mata, J. A.; Peris, E. Chem. Rev. 2009, 109, 3677.

[3] Cho, J.; Hollis, T. K.; Helgert, T. R.; Valente, E. J. Chem. Commun. 2008, 5001.

[4] Bauer, E. B.; Andavan, G. T. S.; Hollis, T. K.; Rubio, R. J.; Cho, J.; Kuchenbeiser, G. R.; Helgert, T. R.; Letko, C. S.; Tham, F. S. Org. Lett. 2008, 10, 1175.

[5] Senthil, A. G. T.; Bauer, E. B.; Letko, C. S.; Hollis, T. K.; Tham, F. S. J. Organomet. Chem. 2005, 690, 5938.

[6] Gu, Z.-G.; Wang, B.-X.; Pang, C.-Y.; Zhou, W.; Li, Z.-J. Acta Chim. Sinica 2012,70, 2501. (顾志国, 王宝祥, 庞春燕, 周文, 李在均, 化学学报, 2012, 70, 2501.)

[7] Zhang, X.-F.; Wright, A. M.; DeYonker, N. J.; Hollis, T. K.; Hammer, N. I.; Webster, C. E.; Valente, E. J. Organometallics 2012, $31,1664$.

[8] Chung, L.-H.; Chan, S.-C.; Lee, W.-C.; Wong, C.-Y. Inorg. Chem. 2012, 51, 8693.

[9] Zhou, H.-J.; Wang, Z.; Gao, C.; You, J.-S.; Gao, G. Chem. Commun. 2013, 49, 1832.

[10] Schlechte, L.; Bon, V.; Grünker, R.; Klein, N.; Senkovska, I.; Kaskel, S. Polyhedron 2012, 44, 179.

[11] Su, Z.; Fan, J.; Okamura, T.; Sun, W.-Y.; Ueyama, N. Cryst. Growth Des. 2010, 10, 3515.

[12] Fan, J.; Gan, L.; Gan, K. H.; Sun, W.-Y.; Yu, K.-B.; Tang, W.-X. Chem. Eur. J. 2003, 9, 3965.

[13] Zhang, S.-Y.; Yang, S.-Y.; Lan, J.-B.; Yang, S.-J.; You, J.-S. Chem. Commun. 2008, 6170.

[14] Wang, H.-Y.; Feng, J.-C.; Jiang, H.-J.; Huang, W.; Wei, W. Prog.
Chem. 2007, 19, 276. (王洪宇, 冯嘉春, 姜鸿基, 黄维, 韦玮, 化 学进展, 2007, 19, 276.)

[15] Wilson, J. N.; Bunz, U. H. F. J. Am. Chem. Soc. 2005, 127, 4124

[16] Spitler, E. L.; Shirtcliff, L. D.; Haley, M. M. J. Org. Chem. 2007, 72,86 .

[17] Davey, E. A.; Zucchero, A. J.; Trapp, O.; Bunz, U. H. F. J. Am. Chem. Soc. 2011, 133, 7716.

[18] Lim, J.; Nam, D.; Miljanic, O. S. Chem. Sci. 2012, 3, 559.

[19] Zhang, L.-Q.; Gu, W.-J.; Chen, C.-X.; Wang, B.-X. Acta Chim. Sinica 2010, 68, 1981. (张林群, 顾玮瑾, 陈长信, 王炳祥, 化学学 报, 2010, 68, 1981.)

[20] Shimizu, M.; Hiyama, T. Chem. Asian J. 2010, 5, 1516.

[21] Luo, J.-D.; Xie, Z.-L.; Lam, J. W. Y.; Lam, C. L.; Chen, H.-Y.; Qiu, C.-F.; Kwok, H. S.; Zhan, X.-W.; Liu, Y.-Q.; Zhu, D.-B.; Tang, B.-Z. Chem. Commun. 2001, 1740.

[22] Hong, Y.-N.; Lamab, J. W. Y.; Tang, B.-Z. Chem. Soc. Rev. 2011, $40,5361$.

[23] Zhang, S.; Qin, A.-J.; Sun, J.-Z.; Tang, B.-Z. Prog. Chem. 2011, 23, 623. (张双, 秦安军, 孙景志, 唐本忠, 化学进展, 2011, 23, 623.)

[24] Available at: http://cbc.arizona.edu/njardarson/group/top-pharmaceuticals-poster.

[25] Li, D.; Gao, B.-J.; Xu, W.-M. Acta Chim. Sinica 2011, 69, 3019. (李 丁，高保娇，许文梅，化学学报, 2011，69, 3019.)

[26] Schwaebel, T.; Trapp, O.; Bunz, U. H. F. Chem. Sci. 2013, 4, 273.

[27] Yu, X.-D.; Liu, Q.; Xu, X.-F.; Lan, H.-C.; Cao, X.-H.; Chen, L.-M.; Liu, B.; Yi, T. Acta Chim. Sinica 2012, 70, 2016. (余旭东, 刘倩, 许秀芳, 兰海闯, 曹新华, 陈黎明, 刘斌, 易涛, 化学学报, 2012 70, 2016.)

[28] Hart, H.; Harada, K.; Frank Du, C.-J. J. Org. Chem. 1985, 50, 3104.

[29] Jian, H.-H.; Tour, J. M. J. Org. Chem. 2003, 68, 5091.

(Zhao, X.) 